\title{
Properties of the Inter-area Modes of the U.S. Eastern Interconnection System
}

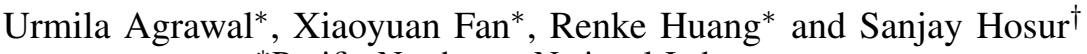 \\ *Pacific Northwest National Laboratory \\ †University of Wyoming \\ Email: Urmila.Agrawal@pnnl.gov
}

\begin{abstract}
With an increase in the oscillation events observed in the U.S. Eastern Interconnection (EI), it has become increasingly important to have a good understanding of the EI system oscillatory behavior. However, as compared to the Western Interconnection system (WI), not much work has been done in this regard for the EI system. Therefore, in this paper, a thorough analysis is carried out to identify inter-area modes and their properties for the EI system using a $80000+$ bus real EI model. Multi-channel Prony method is used in this paper for estimating system modes and mode-shapes.
\end{abstract}

Index Terms-Eastern Interconnection, Modal analysis, Prony method, Small-signal stability Analysis

Power system oscillations are inherent to a power system owing to the system's electromechanical nature such that synchronous machines continuously interact among each other. Most of the times, systems exhibit stable oscillatory behavior and these oscillations damp out quickly in a few cycles. However, under rare circumstances, system oscillations can become unstable resulting in wide-area system outages, as was observed during 1996 WECC blackout [1]. A thorough understanding of system oscillatory behavior is key to avoiding such situations and maintaining reliable system operations.

Low frequency oscillations have been an issue in the Western Interconnection (WECC) system for decades, and therefore the properties of the inter-area modes of the WECC system have been thoroughly studied using both model- and data- based approaches. However, unlike the WECC system, oscillations were not much of an issue for the U.S. Eastern Interconnection (EI) system until recently, and therefore limited work has been done to study the properties of the low frequency oscillations of the EI system.

Some of the studies carried out to understand the oscillatory behavior of the EI system can be found in [2]-[5]. In [2], two EI modes were identified through PMU data for a 2008 Florida oscillation event, having frequencies of $0.22 \mathrm{~Hz}$ and $0.49 \mathrm{~Hz}$ and damping ratio of $15.975 \%$ and with $8.6 \%$, respectively. For the $0.22 \mathrm{~Hz}$ mode, it was observed that the North part of the system was oscillating against the South part of the system. The mode-shape for the second mode could not be identified. This study concluded that the modes of EI were well damped.

In [3], six real-world disturbances that happened in 2008 and 2009 were evaluated. The results showed that the dominant inter-area mode of the EI system having a frequency of 0.20 $0.24 \mathrm{~Hz}$ was observed in the North-West (NW), North-East

This work is supported by the U.S. Department of Energy's Office of electricity through Advanced Grid Modeling program. Pacific Northwest National Laboratory is operated by Battelle for DOE under contract DE-AC0576RL01830.
(NE) and South region of the EI system with NW oscillating against NE and South regions of the EI system. This study also claimed that modes in the EI system are well damped.

In [4], all the wind generators in the EI were classified into four groups, including NW, NE, South, and all remaining ones as the fourth group. The system oscillatory behaviors among NW, NE and South were clearly presented in the simulated generation tripping events, but no further details were provided of those modes and related damping information.

In [5], system modes were estimated based on seven events that occurred in 2016 and 2017. Based on this report, three system modes that were observed are:

- Mode 1: (0.16-0.22 Hz) This mode was observed in two major regions:North and South portions of the EI, primarily the South Atlantic region oscillating against the North-East region.

- Mode 2: (0.29-0.32 Hz) This mode was observed in the same North to South regions as mode 1 with some interactions between the Eastern and Western portions of the EI.

- Mode 3: (0.23-0.24 Hz) For this mode, there were interaction among three regions: Midwest, South Atlantic, and New England.

From these numerous studies, it was observed that the EI modes have generally been well damped with a very complex mode shapes as compared to that of the other interconnections. However, the information obtained from these studies have been limited due to the availability of PMU measurements and the observability of modes at the location of the PMUs. Also, as EI system is much larger than the other interconnection system, a single event cannot excite modes throughout the system. Therefore, measurement-based approaches can only provide limited information on the properties of these interarea modes, such as mode-shape.

With increase of renewable integration and changing operating conditions, it has become increasingly important to have a thorough understanding of the properties of the inter-area modes of the EI system to ensure reliable system operations. In this paper, a detailed study is performed to understand the properties of the inter-area modes of the EI system. The findings of this paper can be used as a benchmark to further advance the understanding of the system oscillatory behavior using real-world measurements. As the damping ratio of system modes vary significantly depending on system operating conditions, the focus of the paper is on mode frequency and their mode-shapes. 
The rest of the paper is organized as follows. Section II briefly describes methodology for obtaining estimates of system modes and their mode-shapes. Section III discusses the EI model used for the analysis and the properties of inter-area modes. Section IV provides the conclusion.

\section{Methodology FOR MODAL ANALYSIS}

This paper performed a detailed model-based analysis to study the properties of the inter-area modes of the EI system. This section briefly describes the methodology used to estimate system modes and mode-shapes, and validate these estimates.

\section{A. Obtaining mode estimates using the Prony method}

In this paper, estimates of system modes given by

$$
\hat{\lambda}_{i}=\hat{\sigma}_{i}+j \hat{\omega}_{i} \quad \forall \quad i=1 \text { to } \mathrm{n},
$$

where $n$ is the number of mode estimates, and the corresponding mode-shape estimates given by a complex variable $\hat{\boldsymbol{B}}_{i}$, were estimated by using multi-channel Prony method described in [6], [7].

\section{B. Validation of system mode estimates}

The estimates of system modes were also validated using approach similar to that described in [8]. For validating mode estimates, the original signal, given by $y[k]$, was compared with the reconstructed signal given by

$$
\hat{y}[k]=\sum_{i=1}^{n} \hat{B}_{i} e^{k \Delta T \hat{\lambda}_{i}} \quad \forall \quad i=1 \text { to } \mathrm{N},
$$

where $\Delta T$ is the data sampling time-period and $N$ is the total number of samples. The goodness of fit error metric is calculated using

$$
G o F=\frac{\|y[k]-\hat{y}[k]\|}{\|y[k]\|}
$$

where $\|$.$\| denotes root-mean-square norm [6]. Based on this$ error metric, model order $n$ was selected which gave the best fit between the original signal and the reconstructed signal.

\section{MODAL ANALYSIS OF THE EI SYSTEM}

In this paper, a 2019 EI PSSE model consisting of 80000+ buses, developed by Multi-regional Modeling Working Group (MMWG) under EI Reliability Assessment Group (ERAG), was used as the base-case to study the properties of the interarea modes of the EI system. As the EI system is quite large, three-phase bus faults were introduced simultaneously at multiple locations, shown in Fig. 1, to generate simulated data. The faults and fault locations were selected such that the generators across several areas of the EI system interacted among each other and resulted in good observability of the modes throughout the system. Also, for identifying the modeshape of system mode estimates, the EI system was divided into a total of sixteen areas based on their geographical location as shown in Fig 3(b).

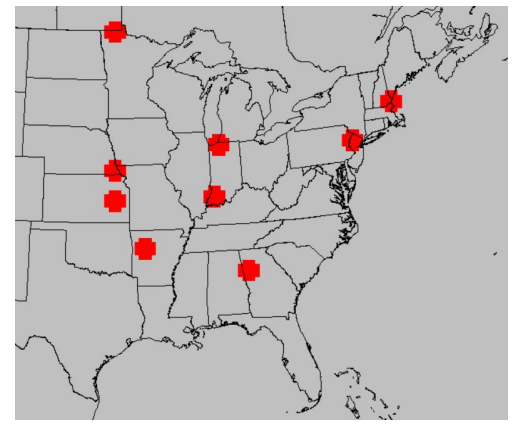

Fig. 1. Locations of the three-phase faults used for generating simulated data

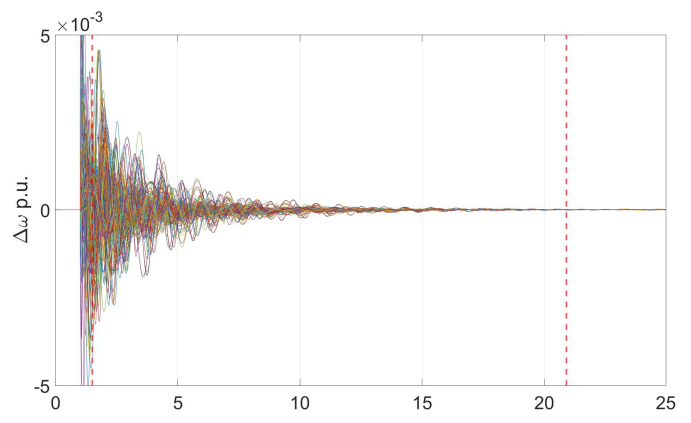

Fig. 2. Generator speed deviation data selected for modal analysis.

TABLE I

INTER-AREA MODE ESTIMATES OBSERVED IN THE SIMULATED DATA

\begin{tabular}{|r|r|r|}
\hline Mode No. & Frequency (Hz) & Damping-ratio(\%) \\
\hline 1 & 0.159 & 30.964 \\
\hline 2 & 0.21 & 29.68 \\
\hline 3 & 0.312 & 19.053 \\
\hline 4 & 0.407 & 12.214 \\
\hline 5 & 0.528 & 12.301 \\
\hline 6 & 0.571 & 9.04 \\
\hline 7 & 0.717 & 8.21 \\
\hline
\end{tabular}

The generator speed deviation data were used for estimating system modes. These simulated data were generated at a sampling rate of 500 samples/sec and were then down-sampled to 5 samples/sec as described in [9]. The multi-channel Prony method was then used to obtain estimates of modes and modeshapes. A total of 15 channels of simulated data were chosen in each area that had the highest root-mean square energy. The average goodness of fit error metric for all channels was less than $1 \%$ validating the estimates of modes and mode-shape for each channel.

Table I provides the estimates of the inter-area modes obtained using the simulated data shown in Fig. 2. The modeshapes are shown for each inter-area mode in Fig. 3 through Fig. 9 using a compass plot and a geographic representation of the EI to show the interactions. In the mode-shape plots using EI map, the area within white-colored circle oscillates against the areas within the red-colored circle. Also, the estimates of first three modes and their mode-shapes match with the estimates described in [5], which were based on real-world disturbances, validating our analysis results. 
Mode-1: Frequency- $0.159 \mathrm{~Hz}$ and Damping ratio- 30.964\%

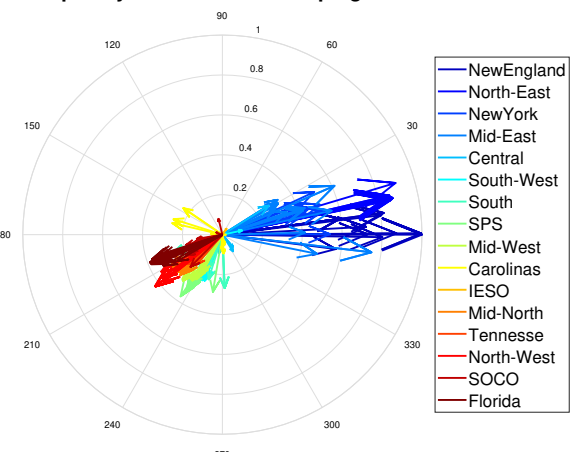

(a) Using compass plot

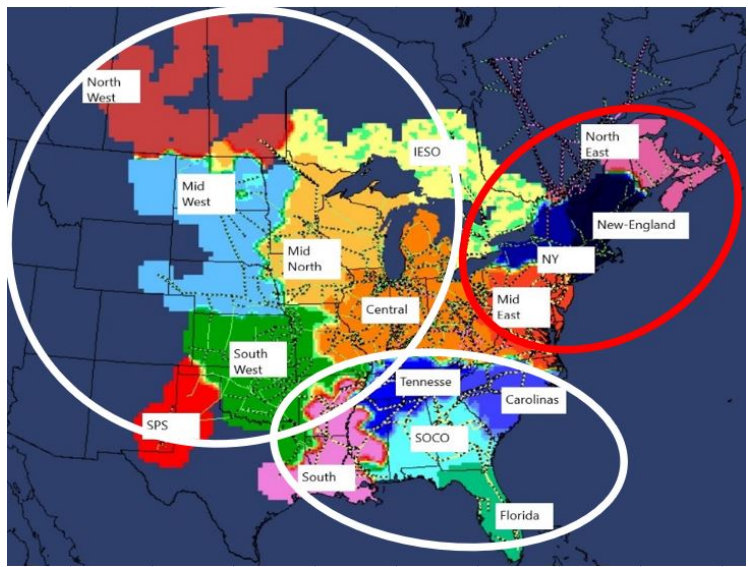

(b) Using geographic representation of the EI

Fig. 3. Mode-shape for $0.159 \mathrm{~Hz}$ mode.

Mode-2: Frequency- $0.21 \mathrm{~Hz}$ and Damping ratio- $29.68 \%$

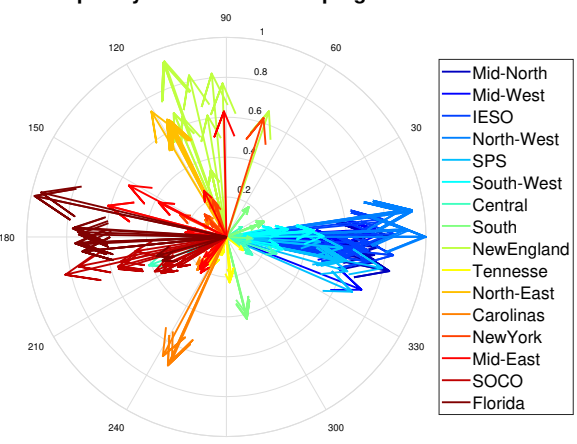

(a) Using compass plot

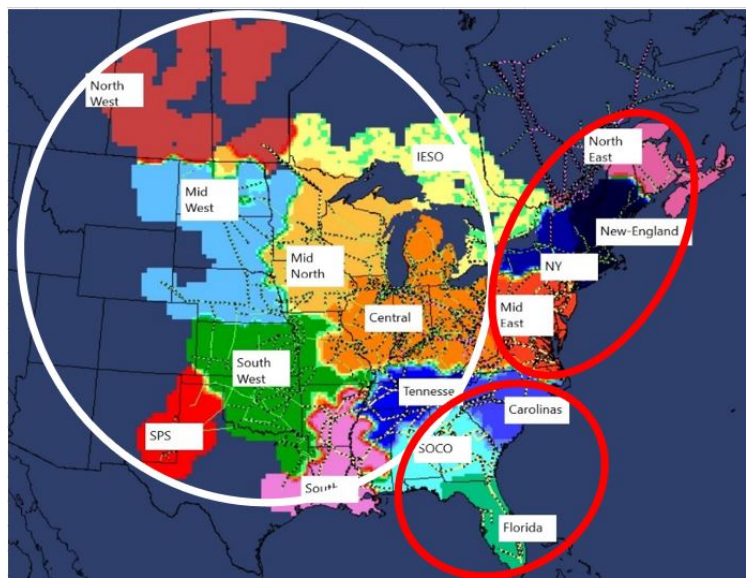

(b) Using geographic representation of the EI

Fig. 4. Mode-shape for $0.21 \mathrm{~Hz}$ mode.

Mode-3: Frequency- $0.312 \mathrm{~Hz}$ and Damping ratio- $19.053 \%$

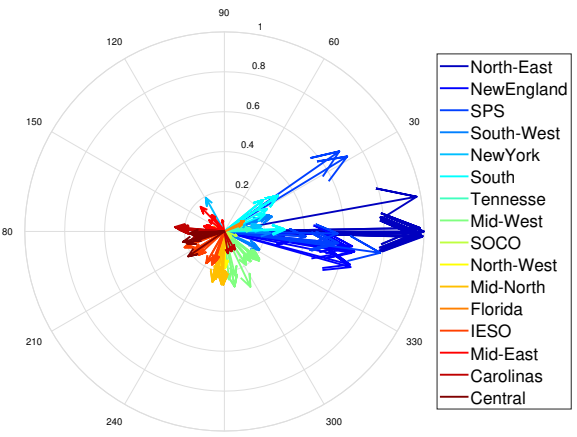

(a) Using compass plot

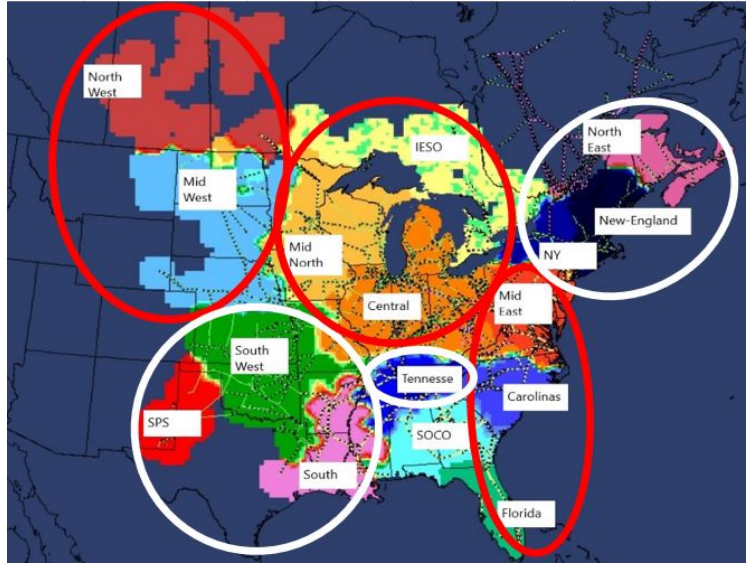

(b) Using geographic representation of the EI

Fig. 5. Mode-shape for $0.312 \mathrm{~Hz}$ mode. 
Mode-4: Frequency- $0.407 \mathrm{~Hz}$ and Damping ratio- $12.214 \%$

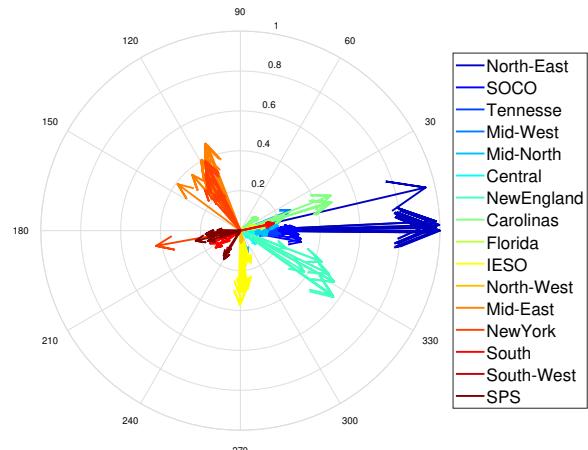

(a) Using compass plot

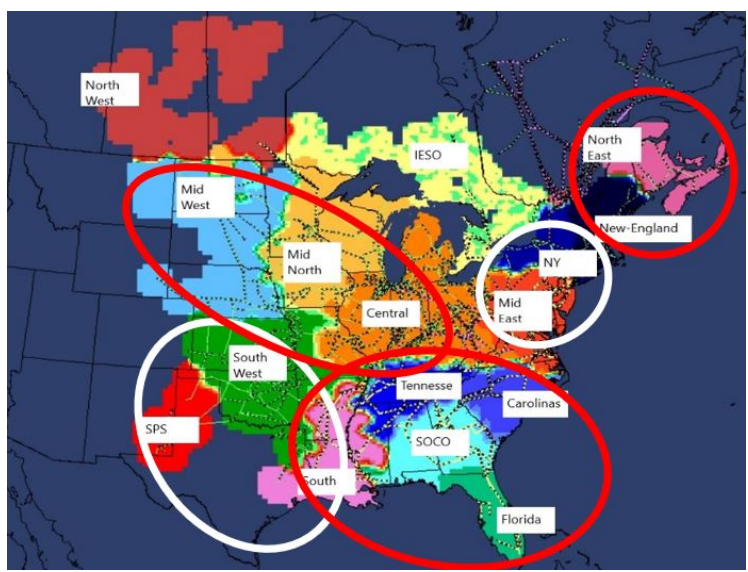

(b) Using geographic representation of the EI

Fig. 6. Mode-shape for $0.407 \mathrm{~Hz}$ mode.

Mode-5: Frequency- $0.528 \mathrm{~Hz}$ and Damping ratio- $12.301 \%$

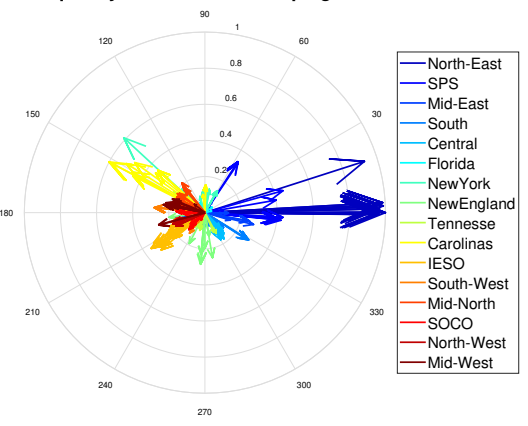

(a) Using compass plot

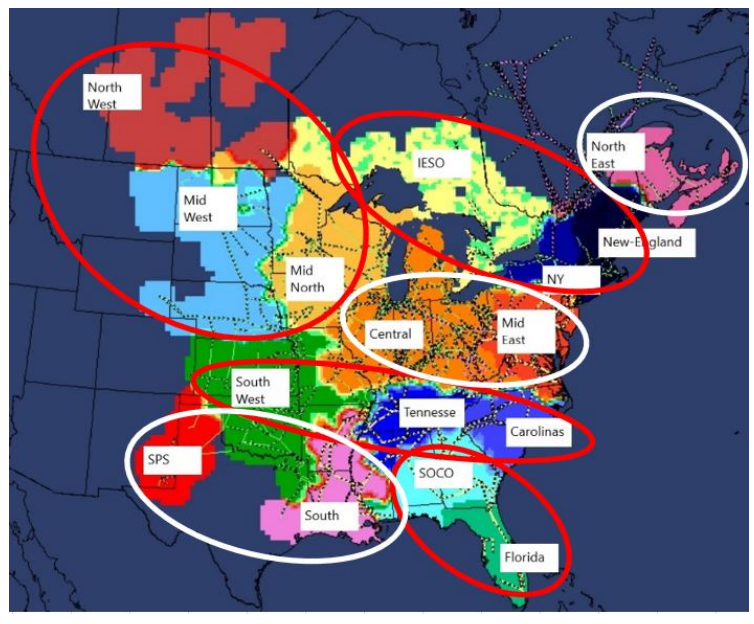

(b) Using geographic representation of the EI

Fig. 7. Mode-shape for $0.528 \mathrm{~Hz}$ mode.

Mode-6: Frequency- $0.571 \mathrm{~Hz}$ and Damping ratio- $9.04 \%$

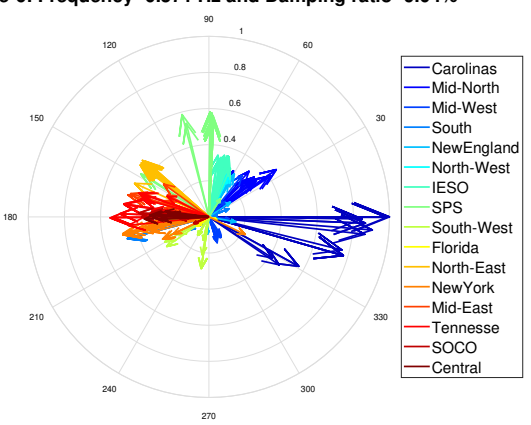

(a) Using compass plot

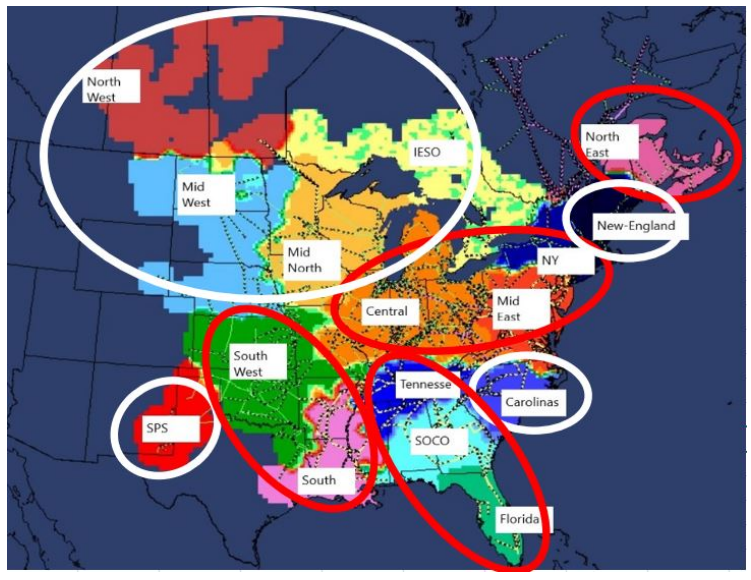

(b) Using geographic representation of the EI

Fig. 8. Mode-shape for $0.571 \mathrm{~Hz}$ mode. 


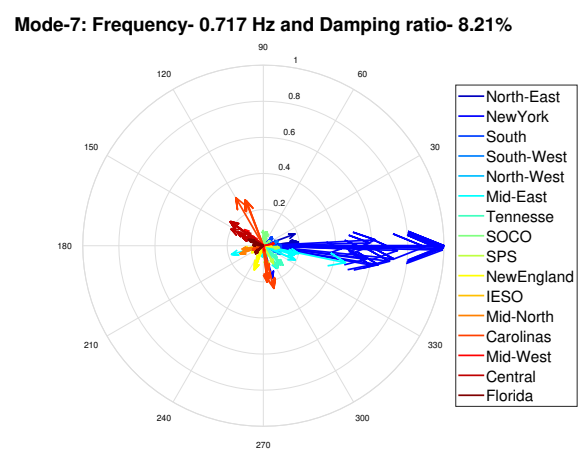

(a) Using compass plot

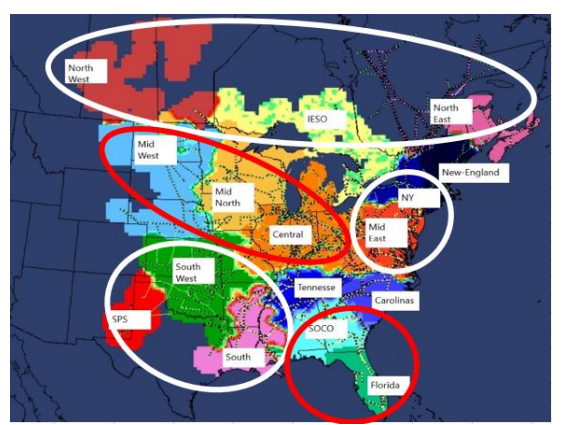

(b) Using geographic representation of the EI

Fig. 9. Mode-shape for $0.717 \mathrm{~Hz}$ mode.

Some observations made for each mode are as follows:

1) $0.159 \mathrm{~Hz}$ mode: For this mode, North-East (NE), New England (NEng), New York (NY) and Mid-East (ME) areas, defined in this paper, oscillated against the rest of the EI system as shown in Fig. 3(b). In 3(a), it was observed that NEng and NE areas have high observability (having normalized amplitude more than 0.75) for this mode, while the observability was good in NY, ME, North-West (NW), Florida (FL), SOCO, SouthWest (SW), South (S), SPS, Mid-West (MW), IESO, Mid-North (MN), and Tennesse (TE) (having normalized amplitude more than 0.2).

2) $0.21 \mathrm{~Hz}$ mode: The mode-shape for $0.21 \mathrm{~Hz}$ mode was similar to that of $0.159 \mathrm{~Hz}$ mode except that SouthEastern part of the EI, that includes FL, SOCO, and Carolinas areas, oscillated with North-Eastern part against the rest of the system as shown in Fig. 4(a). In Fig. 4(b), it was found that the mode has high observability throughtout the EI system across several areas, such as FL, SOCO, NEng, NE, MN, MW, IESO, NW, and SPS.

3) $0.312 \mathrm{~Hz}$ mode: For this mode, the North-Eastern part of the EI system oscillated with the South-Western part against the rest of the system as shown in Fig. 5(b). In Fig. 5(a), it was observed that, NE, NEng and SPS have high observability, while other areas, such as SW, S, MW, NW, MN, IESO, and Central areas have good observability.

4) $0.407 \mathrm{~Hz}$ mode: This mode has a complex mode-shape, as shown in Fig. 6(b), with NEng and NE oscillating against NY and ME. In Fig. 6(a), it was observed that NE have the highest observability, while other areas, such as SOCO, NEng, Carolinas, ME, NY, and SPS have good observability.

5) Other modes: Other three modes having frequencies of $0.528 \mathrm{~Hz}, 0.571 \mathrm{~Hz}$, and $0.717 \mathrm{~Hz}$ have complex modeshapes as shown in Fig. 7(b), Fig. 8(b), and Fig. 9(b), respectively. For mode-5, NE has the highest observability as shown in Fig. 7(a); for mode-6, Carolinas has the highest observability as shown in 8(a); for mode-7, NY has the highest observability as shown in Fig. 9(a).

\section{CONCLUSION}

To conclude, this paper studies the properties of the interarea modes of a real and very complex EI system using a $80,000+$ Bus model. To the authors' knowledge, this level of rigorous study has not been performed before for the EI system. A total of seven inter-area modes were identified; our analysis results obtained for three mode estimates and their mode-shape estimates matched with that obtained using realworld EI PMU data, published in references [2-5], validating our methodology and results. The findings of this paper can be very helpful in understanding the system oscillatory behavior of the EI system and can be used to analyze future oscillatory events taking place in the EI system, including those consisting of forced oscillations. Future work will focus on applying the proposed EI model-based method to study the impact of various high-penetration renewable scenarios on system oscillatory behavior.

\section{REFERENCES}

[1] J. F. Hauer, W. A. Mittelstadt, K. E. Martin, J. W. Burns, and H. Lee, "Interim report of the WECC disturbance monitoring work group," 2005.

[2] L. Vanfretti and J. H. Chow, "Identification of dominant inter-area modes in the Eastern Interconnection from PMU data of the FRCC 2008 disturbance: An eigensystem realization algorithm illustration," 2010.

[3] Z. Yuan, T. Xia, Y. Zhang, L. Chen, P. N. Markham, R. M. Gardner, and Y. Liu, "Inter-area oscillation analysis using wide area voltage angle measurements from FNET," in IEEE PES General Meeting. IEEE, 2010, pp. 1-7.

[4] Y. Liu, J. R. Gracia, T. J. King, and Y. Liu, "Frequency regulation and oscillation damping contributions of variable-speed wind generators in the US Eastern Interconnection (EI)," IEEE Transactions on Sustainable Energy, vol. 6, no. 3, pp. 951-958, 2014.

[5] NERC, "Interconnection oscillation analysis," Jul. 2019. [Online]. Available: https://www.nerc.com/comm/PC/SMSResourcesDocuments/ Interconnection_Oscillation_Analysis.pdf

[6] J. F. Hauer, C. J. Demeure, and L. L. Scharf, "Initial results in Prony analysis of power system response signals," IEEE Transactions on Power Systems, vol. 5, no. 1, pp. 80-89, Feb 1990.

[7] D. J. Trudnowski, J. Johnson, and J. F. Hauer, "Making Prony analysis more accurate using multiple signals," IEEE Transactions on power systems, vol. 14, no. 1, pp. 226-231, 1999.

[8] U. Agrawal and J. W. Pierre, "Visual validation of estimated parametric models of power systems under ambient conditions," in Proceedings of the 52nd Hawaii International Conference on System Sciences, 2019.

[9] J. W. Pierre, D. J. Trudnowski, and M. K. Donnelly, "Initial results in electromechanical mode identification from ambient data," IEEE Transactions on Power Systems, vol. 12, no. 3, pp. 1245-1251, 1997. 